\title{
Archéopages
}

Archéopages

Archéologie et société

Hors-série 2 | 2010

Archéologie sans frontières

\section{La démarche archéo-anthropologique dans la nécropole hellénistique de Plinthine}

\section{Marie-Françoise Boussac et Patrice Georges}

\section{(2) OpenEdition}

1 Journals

Édition électronique

URL : https://journals.openedition.org/archeopages/804

DOI : 10.4000/archeopages. 804

ISSN : 2269-9872

Éditeur

INRAP - Institut national de recherches archéologiques préventives

Édition imprimée

Date de publication : 1 octobre 2010

Pagination : 108-113

ISSN : 1622-8545

\section{Référence électronique}

Marie-Françoise Boussac et Patrice Georges, « La démarche archéo-anthropologique dans la

nécropole hellénistique de Plinthine », Archéopages [En ligne], Hors-série 2 | 2010, mis en ligne le 01 octobre 2010, consulté le 23 février 2023. URL : http://journals.openedition.org/archeopages/804

DOI : https://doi.org/10.4000/archeopages.804 

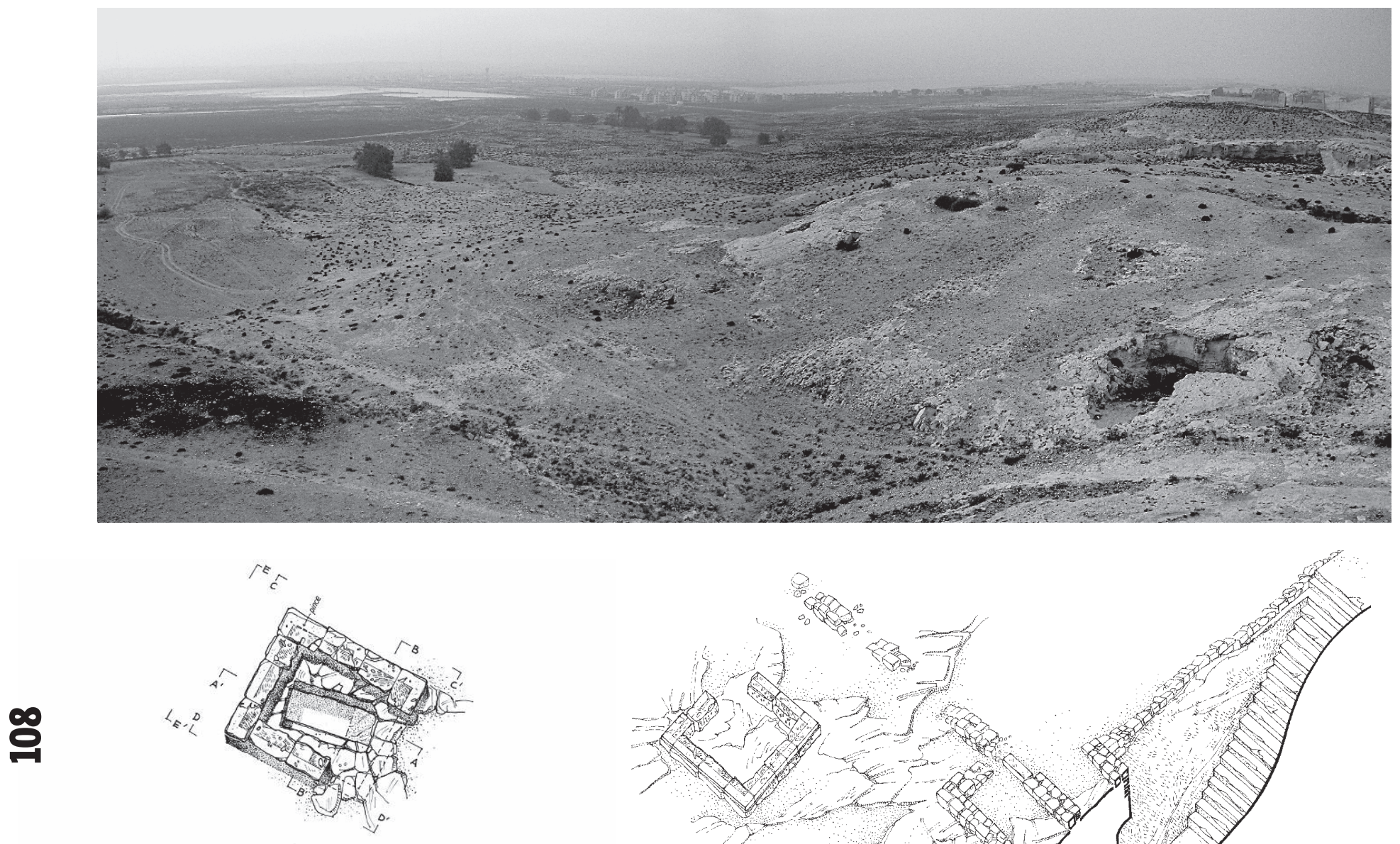

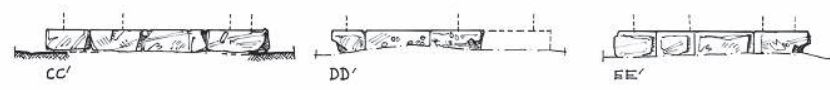
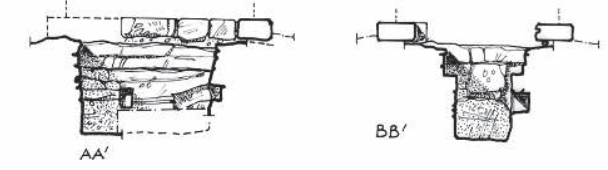

i

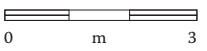

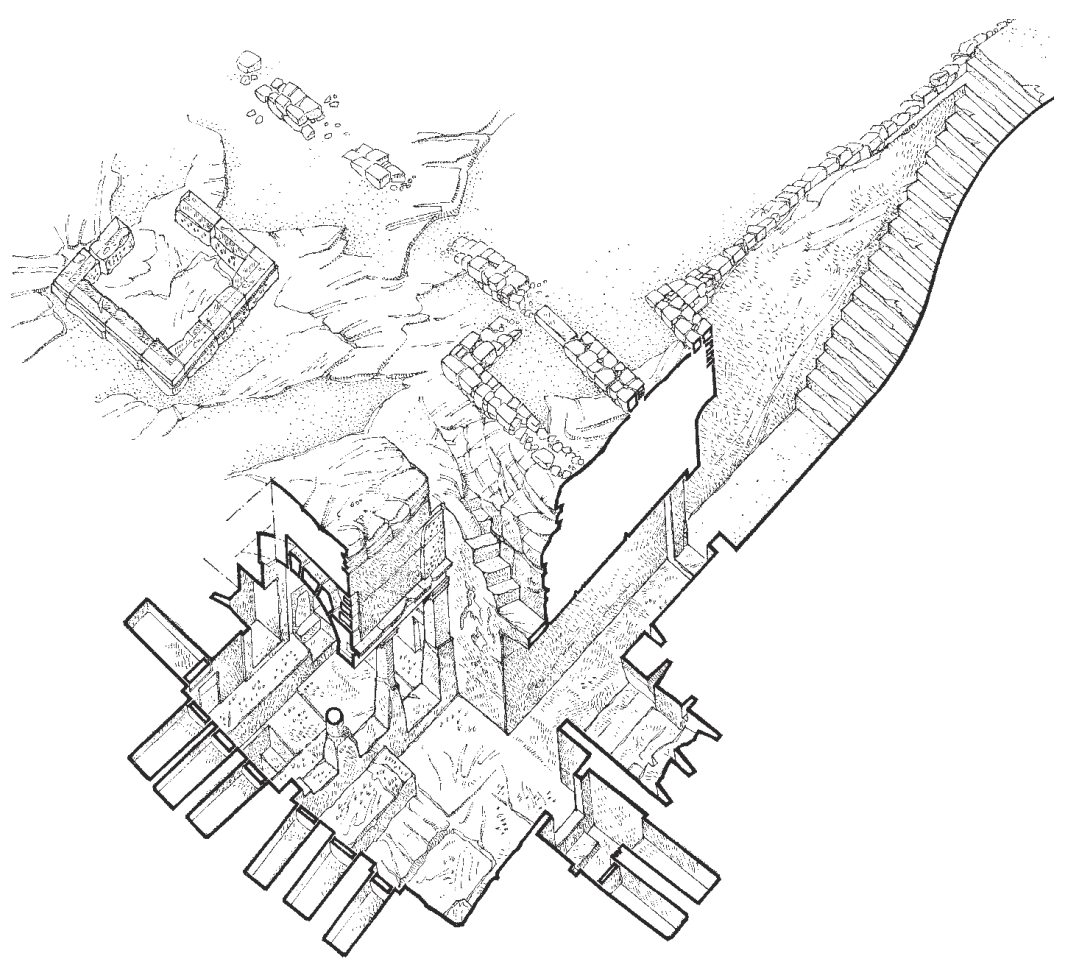

[Fig. 1] Environnement des villes antiques de Plinthine et Taposiris Magna qui fait l'objet des investigations de la MrFTM. Au fond à gauche, le lac Mariout (en cours d'assèchement) et, à droite, les vestiges du temple surplombant la ville de Taposiris Magna.

[Fig.2] Tombe 5o et vestiges du

monument à degrés la signalant.
[Fig.3] Vue axonométrique de la tombe 3 Le dromos de cette tombe permet aux proches de commémorer le défunt. Ce type de tombe rappelle les hypogées alexandrins, dont l'influence serait macédonienne.

[Fig.4] Décor d'un loculus en plâtre particulièrement connu, avec des caractères grecs (chapiteaux des colonnes) et égyptiens (Anubis). Après la conquête grécomacédonienne de l'Égypte, l'installation d'un nombre important d'étrangers, en majorité grecs ou de culture hellène, conduit à l'instauration d'une société multiculturelle.

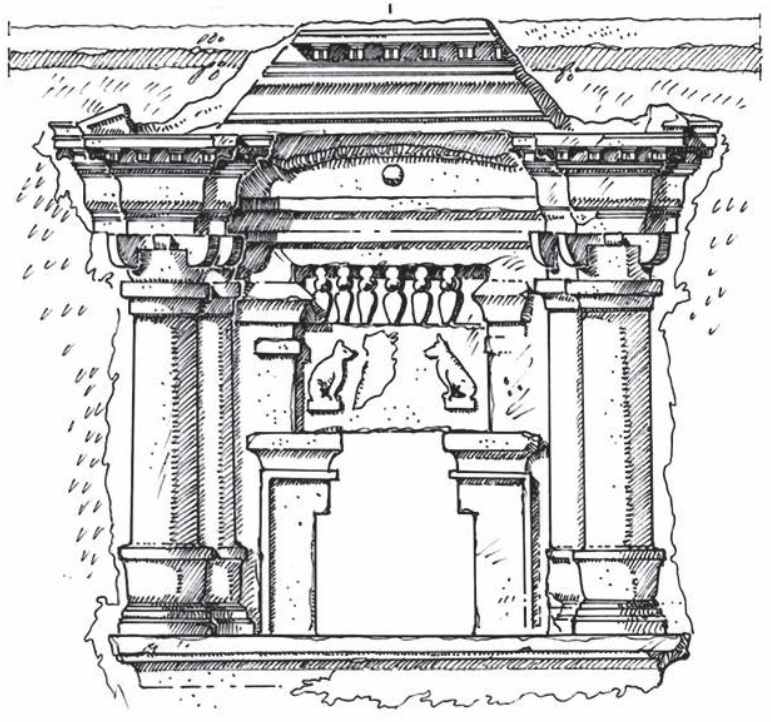


nous renvoie vers nos collections, qu'elle nous pousse à valoriser en les replaçant mieux dans leur contexte, nous pourrions faire bénéficier les Égyptiens de l'expérience française en ce domaine. Nous pourrions aussi leur faire partager notre savoir-faire en matière de protection et de valorisation des monuments historiques. Dans cette optique, Christophe Thiers, le nouveau codirecteur français du Centre franco-égyptien d'étude des temples de Karnak (CNRS-MAEE-CsA) vient de signer avec Mansour Boraïk, codirecteur égyptien du même centre, un accord concernant la formation d'inspecteurs du Conseil suprême des antiquités égyptiennes et celle de jeunes universitaires égyptiens. Les thèmes abordés dans ce chantier-école dérivent de l'étude scientifique des temples de Karnak menée lors des fouilles et autres chantiers conduits par le centre francoégyptien. Jean-Yves Empereur, directeur du Centre d'études alexandrines, fait de même, et son équipe est constituée d'un nombre important d'archéologues égyptien(ne)s.

Dans ces perspectives, la coopération archéologique franco-égyptienne est prometteuse. Par l'intermédiaire de l'ambassade de France au Caire et de missions françaises installées de façon permanente en Égypte, des archéologues de l'Inrap sont de plus en plus souvent sollicités. Ainsi, la mission du Louvre sur le site de Saqqarah va s'enrichir à l'automne 2010 de la collaboration d'un archéologue de l'Inrap, comme le fait déjà sur certains sites l'Institut français d'archéologie orientale du Caire. C'est reconnaître et valoriser la compétence des chercheurs de l'Inrap, leur performance dans l'enregistrement des données archéologiques et l'excellence des résultats qu'ils produisent. Souhaitons à l'archéologie égyptienne, dont la mission la plus urgente est d'être préventive, de voir s'activer des équipes mixtes, dédiées à cette tâche noble et prioritaire.

\section{La démarche archéo- anthropologique dans la nécropole hellénistique de Plinthine}

1 C'est le titre d'une exposition qui, en 1998 avait mis en lumière les vestiges antiques de cette ville et de ses environs.

2 En ce sens, les résultats sont encore plus maigres que dans les nécropoles alexandrine : cf. Dunand, 2002

3 Une opération dirigée par Rached Nouweir en 1954 a fait l'objet d'une courte publication l'anné suivante : Nouweir, 1955. Faisel el-Ashmawy a effectué des recherches de terrain en 1980 . La dernière mission égyptienne, dirigée par Ezet el-Hamamy, a eu lieu en 1982. Ces dernières missions sont inédites. Bref résumé dans Venit, 2002, p. 169-17o. et épargnées par l'urbanisation intensive du littoral méditerranéen : Taposiris Magna et Plinthine (Boussac, 2001 et 2005) [Fig.1]. Leur abandon sans réoccupation, vraisemblablement à la fin de la période hellénistique pour Plinthine et à la fin de l'Antiquité pour Taposiris, a assuré la préservation de leurs vestiges ; certains ont été décrits et figurés par les rares expéditions ayant longé la côte au XIX ${ }^{\mathrm{e}}$ siècle. Remarquées donc, mais éclipsées par la gloire d'Alexandrie ${ }^{\mathbf{1}}$, ces villes sont tombées dans l'oubli, malgré l'intérêt que leur ont porté quelques chercheurs. Elles illustrent pourtant l'urbanisme et les modes de vie des bourgades de la chôra alexandrine. C'est la raison pour laquelle, depuis 1998, la Mission française des fouilles de Taposiris Magna (MғFтM), dirigée par Marie-Françoise Boussac, a engagé l'étude de ces deux sites. Sous l'égide de la Maison de l'Orient Méditerranéen (Mom) et du ministère des Affaires étrangères et européennes, en collaboration avec l'Inrap et avec la participation du Conseil suprême des antiquités égyptiennes, il s'agit d'explorer ces deux cités et leur territoire au rythme annuel d'une mission de terrain.

Fouille ancienne et nouvelles perspectives archéo-thanatologiques pour la nécropole de Plinthine. Parmi les nombreux dossiers développés au sein de la Mfftm, la nécropole de Plinhine n'est pas des moindres. L'importance de cette nécropole pour l'étude des pratiques funéraires a été remarquée par de nombreux auteurs, notamment en raison de son état de conservation (Boussac et al., 2006). En 1937, A. Adriani mène une première campagne de fouilles un an après un pillage limité à quelques tombes. «Toutefois, écrit-il, les recherches que nous avons effectuées permettent un jugement sûr quant aux caractères et à la chronologie de la nécropole, qui (...) garde pour nous toute son importance documentaire pour l'étude des sites. » Il poursuit: « Pour la première fois, dans l'histoire des recherches dans la Maréotis, nous avons trouvé une nécropole de la haute époque hellénistique, ayant toutes les caractéristiques des nécropoles grecques de la métropole » (Adriani, 1952, p. 140). Cet ensemble, que le chercheur juge "vaste », est détaillé au sein d'une publication certes non exhaustive, mais qui autorise aujourd'hui quelques pistes de réflexion à la lumière des découvertes récentes. Les données intéressent essentiellement l'organisation de surface (enclos, monuments funéraires), l'architecture et les pratiques funéraires (inhumation et crémation) (Adriani, 1952) [Fig.2]. Ces dernières sont appréhendées en termes très généraux (cadavres, squelettes et urnes). Ainsi, les précisions concernant les squelettes, pourtant retrouvés par dizaines, sont rares ; aucun examen n'a été pratiqué sur les restes humains.

Le Service des antiquités égyptien a procédé par la suite à d'autres interventions, dans les années 1950 et $1980^{3}$. Chacune de ces fouilles a permis de mettre au jour, plus ou moins accidentellement, des tombes présentant des types très variés allant de la simple fosse (sépulture individuelle de surface)
Marie-Françoise Boussac
Université Paris X-Nanterre, UMR 5189 « Histoire et Sources des Mondes Antiques »

Inrap, UMR 5199 « De la Préhistoire à l'Actuel: Culture, Environnement, Anthropologie»

$\mathrm{D}$ ans l'Antiquité, à l'ouest d'Alexandrie s'étendait la Maréotide, une région prospère en raison de sa proximité avec la capitale des Lagides, mais pas seulement. Bordé au nord par la Méditerranée et baigné par le lac Maréotis au sud, l'endroit était particulièrement propice à la viticulture et au commerce. Un réseau dense de ports lacustres et de villas agricoles constituent les signes les plus évidents de cette situation.

C'est dans ce contexte que se situent deux villes antiques, implantées sur la rive nord du lac Maréotis 

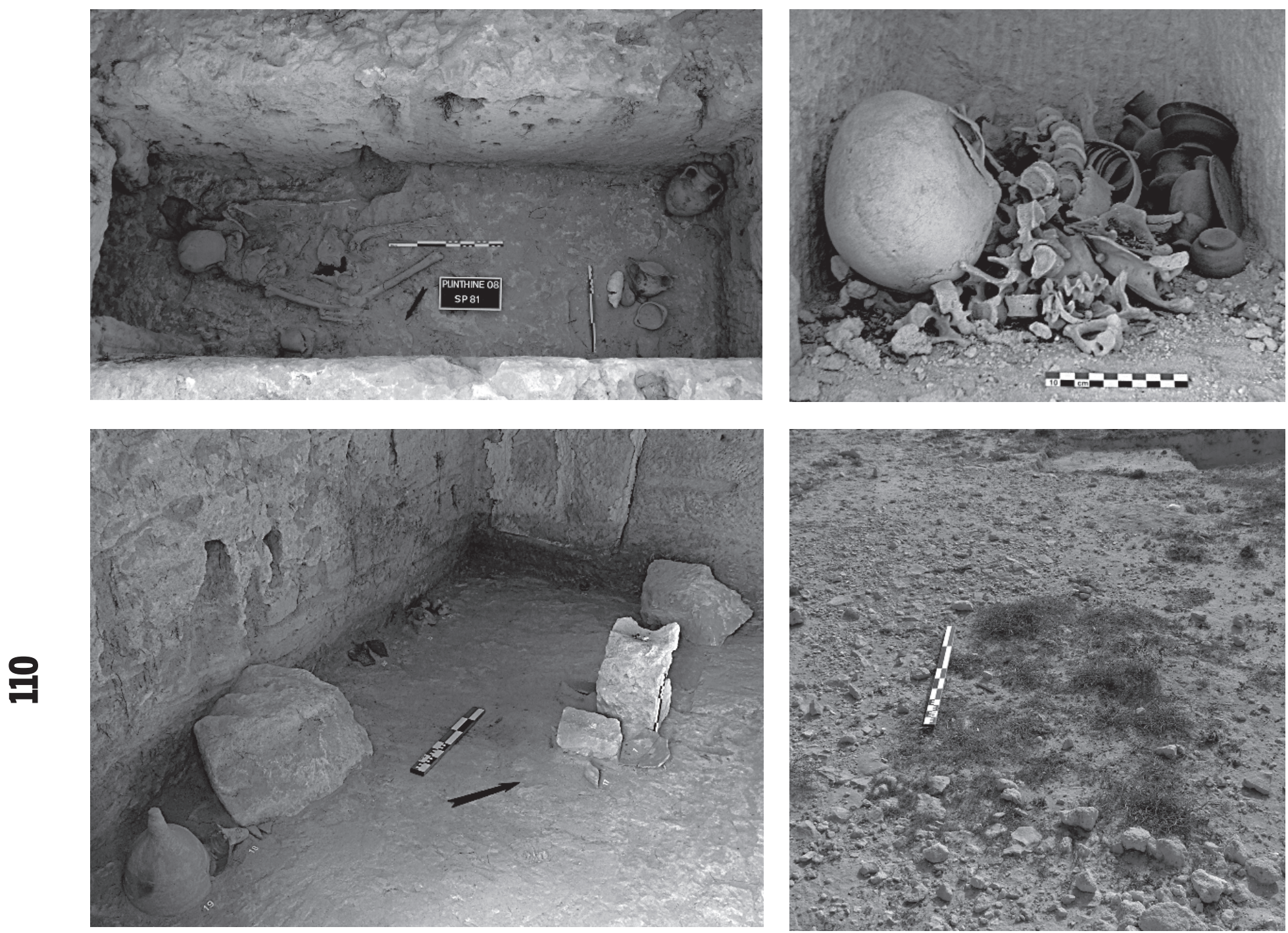

[Fig.5] ] Sépulture 81. Le squelette présente un certain nombre d'anomalies suggérant la disparition d'un élément en matière périssable (support rigide) sur lequel le corps reposait.
[Fig.6] Loculus $5 \mathrm{~A}_{2}$ de la tombe 3 , tel qu'il se présentait à son ouverture.

L'ensemble des artéfacts, datés du début du III ${ }^{e}$ siècle avant notre ère, relève du registre traditionnels des Grecs.
[Fig.7] Artéfacts posés sur le sol de l'unique salle de l'hypogée 77 .

Les tombes souterraines ont été

abandonnées en létat, sans doute au moment de la désaffection de la ville de Plinthine en faveur de Taposiris Magna. On observera, sur la paroi, la trace laissée par le comblement (éolien) de la salle, de l'entrée vers le fond.
[Fig.8] L'approche stratigraphique a permis de mettre en évidence les particularités phytographiques du site. Sur la base de ces indices, nous avons retrouvé dans les sépultures de surface (ici la sépulture 86) des ouvertures d'hypogées. 
à des hypogées à dromos avec une ou plusieurs chambres [Fig.3]. Les modalités de découverte des sépultures et loculi - des niches taillées dans la paroi des hypogées pour y déposer les défunts montrent que les squelettes, quand ils étaient prélevés, n'étaient pas une priorité, voire un sujet d'étude. Quoi qu'il en soit, le potentiel archéologique de cette nécropole est confirmé (Nouweir, 1955). Il s'avère en effet qu'elle a été abandonnée à la fin de l'époque hellénistique et qu'elle n'a jamais été réutilisée. Elle est une source de première importance sur les pratiques et les mentalités des premières générations de Grecs installés en Égypte [Fig.4]. L'évolution de leurs coutumes en Maréotide ne pouvait être mesurée qu'à l'aide d'une étude archéo-anthropologique adaptée, au centre de laquelle le corps, étudié in situ, tient une place prépondérante.

Pour cette partie de l'Égypte, comme pour la période considérée, cette approche était loin d'être évidente. En effet, dans l'esprit de chacun, l'Égypte est l'endroit où la conservation des vestiges en matière périssable est optimum. Mais dans la région d'Alexandrie, le total pluviométrique annuel est relativement important. Les vents marins maintiennent par ailleurs un taux d'humidité relative moyen, voire haut, et constant qui explique la disparition, totale ou presque, des vestiges organiques, d'une façon comparable à ce qui se produit en France [Fig.5]. De fait, les contextes plus favorables ont indéniablement été favorisés par les recherches (Haute-Égypte, milieux désertiques, etc.) et les artéfacts accompagnant le corps, ou les études architecturales, ont pris une part dominante de la réflexion sur les gestes et pratiques funéraires [Fig.6]. Il était donc important, sans abandonner ces approches, d'intégrer pleinement une vision archéo-thanatologique dans l'étude de la nécropole de Plinthine. D'autant plus que la MFFTM s'appuyait, à ce moment-là, sur l'expérience réussie de l'intervention d'une équipe d'archéo-anthropologues, composée essentiellement d'agents de l'Afan puis de l'Inrap sur le site du Pont de Gabbari à Alexandrie (Empereur, Nenna, 2001; Empereur, Nenna, 2002). Cette fouille préventive, menée dans les quartiers ouest de la ville, avait en effet déjà permis d'engager une réflexion méthodologique quant aux difficultés de fouiller d'importants ensembles souterrains. Ces tombes, si monumentales soient-elles, sont généralement comblées, tout du moins en partie. Ce qui diffère des tombes pharaoniques où, une fois l'entrée découverte, tout l'intérieur se trouve d'emblée visible par l'inventeur [Fig.7].

Mettre en évidence ce qui n'apparaît pas, se concentrer in situ, quelles que soient les conditions, sur la position des ossements humains, gérer les différents aspects archéologiques de la nécropole, même s'il ne s'agit pas de tombes, sont autant de missions dont les archéo-anthropologues de l'Inrap sont chargés sur l'ensemble du territoire métropolitain, mais aussi ailleurs. À ces tâches, sans doute faut-il ajouter, comme tous les agents de cet Institut, l'habitude de s'adapter à des

terrains différents, voire difficiles, et la faculté d'approcher les caractéristiques d'un tel site à partir de sondages. Bref, autant de raisons qui justifiaient d'accorder une place importante à un archéo-anthropologue de l'Inrap dans le cadre de cette recherche pluridisciplinaire et pluri-institutionnelle.

Deux pistes, qui n'avaient pas été vraiment suivies par nos prédécesseurs, ont été envisagées conjointement ; elles découlent du modus operandi de l'archéologie préventive des ensembles funéraires: 1/ appréhender la nécropole d'un point de vue chronologique et spatial (délimitation, analyse de sa formation, son développement, etc.) et 2/ éclairer les gestes et les comportements funéraires (Georges, 2002). Les apports de cette démarche ont été évidents.

\section{Résultats des campagnes de fouille sur} les pratiques funéraires. À la manière d'une opération de diagnostic, quelques sondages ont permis rapidement de cerner l'emprise de cet ensemble. Ils ont non seulement permis de révéler des hypogées inédits, mais également des traces d'occupation, en dehors de la surface estimée (et protégée) jusqu'alors. À l'ouest, la fin de la nécropole est marquée par des tas plus ou moins importants de déchets de taille, dont le sommet affleure parfois aujourd'hui. Il apparaît donc que la nécropole en surface avait manifestement tout d'un chantier, loin de l'image idéale de la nécropole antique. Ces tas font d'ailleurs écho à d'autres retrouvés ici et là, dans l'environnement immédiat des tombes. Ils nous ont permis d'aborder une chronologie relative du site, certains tas condamnant l'accès à des tombes.

La plupart des tombes sont situées au sommet d'une colline, la taenia des auteurs antiques ; le rocher (aujourd'hui) apparent dans la zone sommitale a en effet facilité l'installation des tombes (taillées). Cet état de fait n'a poussé aucun des auteurs à chercher des traces d'architecture périssable, visibles si l'on applique une fouille stratigraphique. C'est le cas de certains murs de terre crue qui n'avaient pas attiré l'attention des chercheurs précédents, tant il est vrai que les techniciens dits de fouille ne sont généralement pas formés à dégager des couches. C'est la raison pour laquelle nous avons initié empiriquement les ouvriers de fouille vivant à proximité du site, ainsi que les inspecteurs du Conseil supérieur des antiquités égyptiennes intéressés par les méthodes mises en œuvre couramment à l'Inrap.

Hormis les innombrables couches de sables (apport éolien), qu'il demeure difficile d'individualiser compte tenu de leur épaisseur - ce qui n'aurait de toute façon pas de pertinence archéologique -, la stratigraphie rend compte d'un phénomène de concrétion, lié à la pédogénèse et à l'apport d'eau météoritique affectant le sol riche en carbonates. Considérée par d'aucuns comme le substrat, cette croûte, selon son épaisseur constatée, s'est avérée en fait être un bon indice pour la découverte des nouveaux hypogées, tout aussi importants que les indices phytographiques [Fig.8]. Non reconnu comme tel, cet encroûtement calcaire de couleur les plus récents, il n'est races pérennes, La question se pose les sépultures d'individus À paraître).

5 Ainsi, pour les sépultures de surface, il n'est pas rare clacée à l'emplacement présumé des pieds, là où se concentrent généralement les offrandes. 

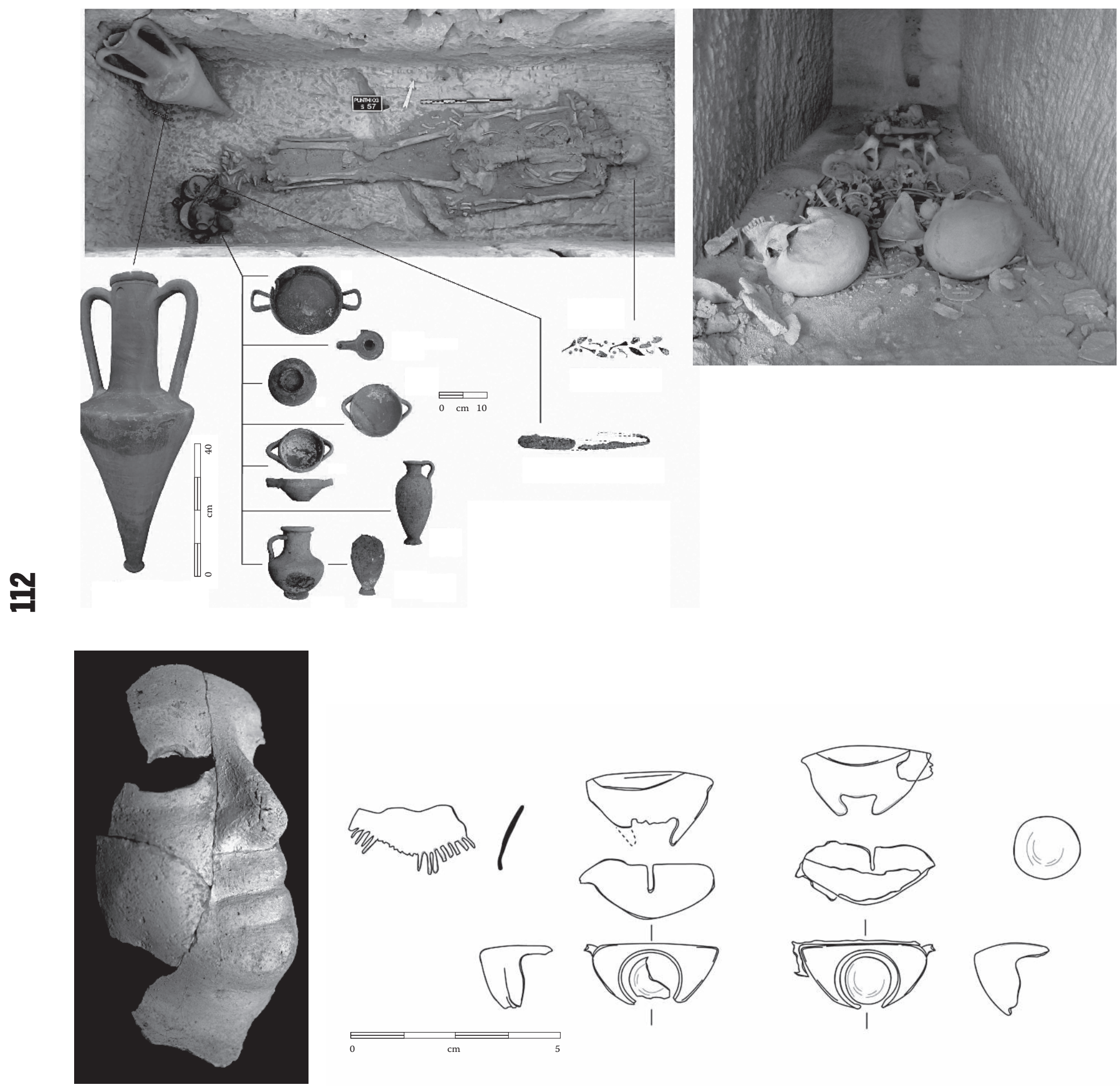
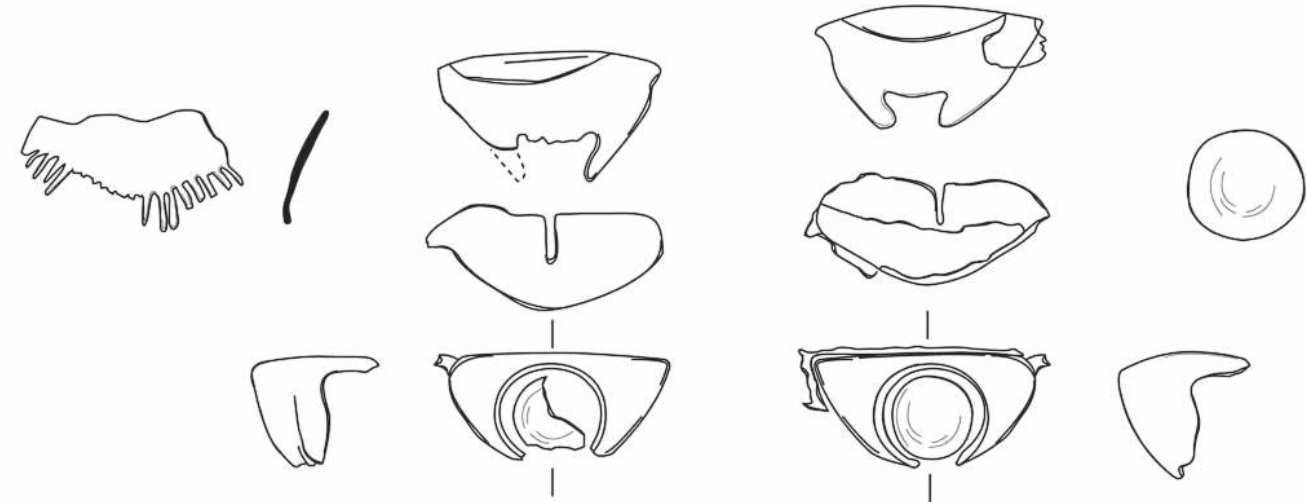

[Fig.9] Létude de la position des os et des artéfacts de la sépulture 57 permet d'envisager une

décomposition en espace vide. Cela amène à s'interroger sur plusieurs anomalies, telles que la position de la clavicule droite et celle du crâne (par rapport aux éléments de la couronne). La disparition de vestiges organiques est illustrée par les éléments épars de la couronne de bronze (qui devait être initialement maintenue sur la tête), des traces de tissus sur le strigile et des offrandes imbriquées, mais sans contact physique.
[Fig. 10] Vue du loculus 6D2 de la tombe 3 , juste après l'ouverture. Au premier plan, à gauche, les pierres et fragments de plâtre révèlent des ouvertures multiples ; on aperçoit, à droite, des fragments de plâtre d'un masque funéraire dans l'environnement immédiat du crâne.
[Fig. 11] Plusieurs masques funéraires ont été retrouvés dans les loculi de la tombe 3. Ils sont tous à l'état de fragments. Leur position permet de les attribuer à tel ou tel individu, voire de les associer à des éléments de décor singuliers.
[Fig. 12] Quand la disparition du masque funéraire est complète, comme dans ce cas de l'hypogée 52 , il reste les yeux en pierre. Notons que, sur d'autres sites, une paire d'yeux identique avait été attribuée à une statue. Les découvertes de Plinthine tendent à montrer qu'il s'agit en fait d'éléments de masques funéraires. 
blanche a protégé les vestiges archéologiques dans les parties non fouillées de la nécropole.

L'étude archéo-anthropologique de cette nécropole vise surtout à redonner voix «aux plus nombreux » : l'objectif était de définir l'attitude du groupe inhumant vis-à-vis des défunts dont il s'est occupé avec plus ou moins de précaution, voire d'ostentation. Or les interventions archéologiques précédentes n'ont laissé aucune information de ce type, pas même sous la forme rudimentaire de documents photographiques. Cette nécropole, comme bien d'autres, a en effet largement souffert d'une approche archéologique centrée sur l'objet. De telle sorte que, comme l'a souvent dit Henri Duday, on pourrait croire que le squelette, rarement enregistré, souvent laissé in situ à Plinthine, accompagnait les offrandes... et non l'inverse ! Tant il est vrai que la découverte d'un squelette a souvent été considérée comme une gêne, l'objet prenant alors le pas sur le défunt ou ce qu'il en reste. La description et le prélèvement des squelettes ont donc été orientés selon les modalités de l'archéo-thanatologie funéraire (Duday, 2005; Duday, 2006; Duday, Guillon, 2006). Comme pour les opérations préventives en France, cela revient à reconnaître les distorsions éventuelles des facteurs taphonomiques et humains par rapport à l'agencement initial de la sépulture. À la différence près qu'il ne s'agit pas de déterminer les incidences du milieu de décomposition : les loculi sont des espaces vides retrouvés comme tels. Mais des éléments liés au corps ou des gestes funéraires, imperceptibles si l'attention n'est pas portée sur le squelette in situ, peuvent entraîner des distorsions dans l'agencement du squelette. Pour ce site, comme pour d'autres de la région alexandrine, les observations ostéo-archéologiques pallient la disparition de la matière organique (Georges et al., 2003) [Fig.9 et 10]. Les os, selon leur position (au sein de la sépulture et les uns par rapport aux autres), apportent en premier lieu des renseignements archéologiques sur la tombe, avant même de faire l'objet d'analyses paléobiologiques. Le corps est de facto replacé au cœur de la discussion, quelle que soit la richesse de la tombe dont l'étude s'avère globale. Grâce à une telle approche, nous avons révélé des éléments initialement placés sur ou autour du corps, mais aujourd'hui disparus.

Ce qui est valable pour les ossements l'est également pour les objets. L'analyse de mouvements éventuels du fait de la décomposition du corps contribue également à préciser le contexte de dépôt. Cette méthodologie permet aussi de discuter, sinon de la nature, tout du moins de la position de certains artéfacts initialement au contact du corps. C'est ainsi le cas des éléments de décoration de masques funéraires, seuls ceux étant parfaitement conservés dans les musées ayant fait l'objet d'études [Fig.11 et 12]. Ce qui a pu créer un biais typo-chronologique.
Adriani A., 1952, « Nécropole et ville de Plinthine », Annuaire du Musée gréco-romain, III, p. 140-159.

Boussac M.-F., 2001, « Deux villes de Maréotide : Taposiris et Plinthine », Bulletin de la Société française d'Égyptologie, 150, p. 42-72.

Boussac M.-F., 2005, «Taposiris Magna et Plinthine », in Archéologies. Vingt ans de recherches françaises dans le monde, Paris, Maisonneuve et Larose, p. 347.

Boussac M.-F., Georges P., Zouair N., 2006, « Égypte : un hypogée intact à Plinthine ", Archéologia, 431, p. 8-9.

Duday H., 2005, « L'archéothanatologie ou l'archéologie de la mort », in Dutour O., Hublin J.-J., Vandermeersch B. (ÉD), Objets et méthodes en paléoanthropologie, Comité des Travaux Historiques et Scientifique, Orientations et méthodes, n 7, Paris, p. 153-215.

Duday H., 2006, « L'archéothanatologie ou l'archéologie de la mort (Archaeoethnoanatology or the Archaeology of Death) ", in Gowland R., Knüssel C., Social Archaeology of Funerary Remains, Oxford, Oxbow books, p. 30-56.

Duday H., Guillon M., 2006, « Understanding the Circumstrances of Decomposition when the Body Is Skeletonized », in SснмітT A., Cunha E., Pinheiro J., Forensic Anthropology and Medecine. Complementary Sciences From Recovery to Cause of Death, Totowa, New Jersey, Humana Press, p. 116-157.

DunAND F., 2002, « De la cendre à la myrrhe. Les usages du corps mort en Égypte tardive », in LABRIQUE F. (ÉD.), Religions méditerranéennes et orientales dans l'Antiquité, Le Caire, Bibliothèque d'Étude, 135, p. 101-119.

Empereur J.-Y., NenNa M.-D. (ÉD.), 2001, Nécropolis 1, Études alexandrines 5, Le Caire, Ifao, 527p.

Empereur J.-Y., Nenna M.-D. (ÉD.), 2003, Nécropolis 2, Études alexandrines 7, Le Caire, Ifao, 714p.

Georges P., À paraître, « Où sont les enfants de la nécropole hellénistique de Plitnthine? ", in Types de tombes et traitement des enfants dans l'antiquité gréco-romaine, Actes du colloque d'Alexandrie 12-14 novembre 2009.

GeORGES P., 2002, « Les pratiques funéraires de la nécropole hellénistique de Plinthine : résultats préliminaires ", in CHARON A. (DIR.), La mort n'est pas une fin. Pratiques funéraires en Égypte d'Alexandre à Cléopâtre, Catalogue de l'exposition 28 septembre 2002-5 janvier 2003, Arles, Musée de l'Arles antique, p. 72-74.

Georges P., BoËs E., Alix G., Schmitt A., 2003, « Des momies éphémères et des os pour l'éternité. La gestion paradoxale de la Necropolis d'Alexandrie à l'époque romaine ", in BALLET P., Cordier P., Dieudonne-Glad N. (Dir.), La ville et ses déchets dans le monde romain : rebuts et recyclages, Actes du colloque de Poitiers (19-21 septembre 2002), Montagnac, Éditions Monique Mergoil, p. 277-301.

Maureil B., Sellier P., 1996, « Dislocation en ordre paradoxal, momification et décomposition : observations et hypothèses », in Castex D., Sellier P., Duday H., Bruzek J. (éd.), Méthodes d'étude des sépultures, du terrain à l'interprétation des ensembles funéraires, Paris, Bulletins et Mémoires de la Société d'Anthropologie de Paris, t. 8, n 3-4, p. 313-327.

Venit M., 2002, Monumental Tombs of Ancient Alexandria. The Theatre of the Dead, Cambridge, University press.

\section{Collaboration franco-israélienne dans la recherche archéologique}

Hamoudi Khalaily

Israel Antiquities Authority

$\mathrm{D}$ epuis le début du $\mathrm{xx}^{\mathrm{e}}$ siècle, lorsque la recherche archéologique en Israël a été établie, les délégations étrangères venues d'Europe et des États-Unis ont collaboré avec les institutions locales. Les savants français ont été des pionniers en ce domaine. René Neuville, par ses fouilles sur de nombreux sites néolithiques et mésolithiques, a été particulièrement actif dans le développement de l'archéologie israélienne. Ses études ont eu une influence majeure sur l'institutionnalisation de l'étude de la Préhistoire du sud du Levant et ses recherches coïncidaient avec celles de l'institut archéologique de l'Université hébraïque à Jérusalem. Son disciple et successeur, Jean Perrot, a créé la délégation permanente française à Jérusalem et a collaboré avec plusieurs 